\title{
CARCINOMA DIFERENCIADO DA TIREÓIDE: A VALIDADE DA COMPLEMENTAÇÃO DA TIREOIDECTOMIA
}

\author{
DIFFERENTIATED THYROID CANCER: \\ THE VALUE OF COMPLETION OF THYROIDECTOMY
}

\author{
José Luís Braga de Aquino, TCBC ${ }^{1}$ \\ José Gonzaga Teixeira de Camargo ${ }^{1}$ \\ Celso Müller Bandeira ${ }^{1}$ \\ José Francisco Salles Chagas ${ }^{2}$ \\ Alexandre Yamashita ${ }^{3}$ \\ Eduardo Pereira ${ }^{3}$
}

\begin{abstract}
RESUMO: Objetivo: Analisar a invasão tumoral do lobo contralateral da glândula tireóide no carcinoma diferenciado, correlacionando o risco/benefício com as complicações decorrentes de uma segunda intervenção. Método: De outubro/93 a dezembro/96 foram operados 20 pacientes com carcinomas diferenciados da glândula tireóide. Os parâmetros analisados foram sexo, idade, tipo de operação, tipo de complicações, histopatológico da peça cirúrgica e invasão do lobo contralateral. Eram dois pacientes do sexo masculino (10\%) e 18 do feminino (90\%); as idades variaram de 17 a 89 anos; o tipo histológico mais freqüente foi o carcinoma papilífero (13 casos), seguido do folicular (seis casos) e carcinoma de células de Hürthle (um caso). Como primeiro procedimento cirúrgico houve 11 lobectomias + istmectomias, quatro lobectomias subtotais e uma istmectomia. Cinco pacientes não realizaram a totalização (um por fibrose, três por perda de seguimento e um por ser microcarcinoma). Resultados: Na análise do lobo contralateral realizada em 15 pacientes, 11 resultaram negativas e outras quatro positivas $(26,6 \%)$. As complicações apresentadas foram rouquidão (dois casos revertidos com tratamento fonoterápico), hipoparatireoidismo (dois casos, um transitório e um permanente). Conclusão: A totalização da tireoidectomia é um procedimento importante no tratamento do tumor maligno da tireóide pela alta porcentagem de metástase contralateral $(26,6 \%)$. Além disso, é um procedimento com mortalidade nula e pequena incidência de complicações.
\end{abstract}

Descritores: Carcinoma de tireóide; Cirurgia.

\section{INTRODUÇÃO}

O tratamento cirúrgico de escolha no câncer diferenciado da tireóide sempre foi motivo de controvérsias. Alguns advogam a lobectomia unilateral e istmectomia por evidenciarem não haver diferença na sobrevida, quando comparadas com a tireoidectomia total, além da diminuição da morbidade pós-operatória ${ }^{1-12}$. Outros ainda têm demonstrado as vantagens da tireoidectomia subtotal no tratamento do carcinoma bem diferenciado, removendo todo o lobo afetado, istmo e parte do lobo contralateral, pois levaria à diminuição da taxa de recorrência local quando comparada aos pacientes submetidos ao procedimento unilateral $^{13,14}$.
O problema se torna ainda maior quando se evidencia no paciente que é submetido à lobectomia unilateral, por um nódulo tireoidiano e o diagnóstico de câncer só é confirmado após a operação. Isto pode ocorrer pela limitação da biópsia de congelação intra-operatória, seja pela dificuldade de realização da mesma ou mesmo em diferenciar lesões benignas de malignas, nas lesões foliculares ${ }^{15-17}$.

Assim, a lobectomia unilateral, realizada para o provável nódulo benigno, pode demonstrar carcinoma diferenciado da tireóide nos achados anatomopatológicos alguns dias após a intervenção inicial, o que significa para aqueles autores favoráveis à resecção total da glândula, uma outra operação que seria a totalização tireoidectomia ${ }^{3,5,6,8,10,15,18-}$ ${ }^{21}$. Por sua vez, alguns argumentam que a reoperação au-

1. Assistente do Serviço.

2. Chefe do Serviço.

3. Estagiário do Serviço.

Recebido em 8/11/1999

Aceito para publicação em 26/12/2000

Trabalho realizado no Serviço de Cirurgia de Cabeça e Pescoço do Hospital e Maternidade Celso Pierro da Pontifícia Universidade Católica de Campinas. 
mentaria muito a morbidade, o que não justificaria tal procedimento no câncer diferenciado da tireóide ${ }^{22}$.

Na literatura nacional, os dados referentes à remoção do tecido tireoidiano remanescente pela complementação da tireoidectomia na segunda operação são limitados, não havendo nenhum trabalho que analise de maneira mais específica este aspecto.

Assim sendo, o objetivo deste estudo é analisar os resultados da complementação da tireoidectomia em uma série de pacientes com carcinoma diferenciado da tireóide unilateral e avaliar as potenciais complicações que poderiam ocorrer pela segunda operação.

\section{MÉTODO}

No período de outubro de 1993 a dezembro de 1996, no Serviço de Cirurgia de Cabeça e Pescoço do Hospital e Maternidade Celso Pierro da Pontifícia Universidade Católica de Campinas, foram operados 20 pacientes com bócio tireoidiano.

Com relação ao sexo, 18 pacientes eram do sexo feminino (90\%) e dois do masculino (10\%); a idade foi variável de 17 a 89 anos, com média de 45 anos.

Na primeira intervenção foram realizadas 15 lobectomias com istmectomia por apresentarem nódulo sólido ao exame de ultra-sonografia cervical pré-operatório. Em quatro pacientes houve tireoidectomia subtotal por apresentarem bócios multinodulares em ambos os lobos tireoidianos e o paciente restante foi submetido somente à istmectomia, por apresentar nódulo único no istmo (Tabela 1).

Tabela 1

Número de pacientes, segundo a técnica cirúrgica da primeira intervenção operatória

\begin{tabular}{l|c|c}
\hline Técnica operatória & $\begin{array}{c}\text { No de } \\
\text { pacientes }\end{array}$ & $\%$ \\
\hline Lobectomia com istmectomia & 15 & 75 \\
Tireoidectomia subtotal & 4 & 20 \\
Istmectomia & 1 & 5 \\
\hline
\end{tabular}

Em relação ao tipo histopatológico da peça cirúrgica, o mais freqüente foi carcinoma papilífero, presente em 13 casos (65\%), seguido pelo carcinoma folicular e carcinoma de células de Hürtle (Tabela 2). Em todos esses pacientes foi indicada a totalização da tireoidectomia, em um período variável de 15 a 30 dias após a primeira intervenção cirúrgica.

\section{RESULTADOS}

Dos 20 pacientes estudados, em cinco (25\%) não foi realizada a totalização da tireoidectomia; três recusaram a realização da segunda intervenção, com perda do seguimento dos mesmos; em um havia intensa fibrose tecidual no campo cirúrgico e outro por se tratar de microcarcinoma.
Tabela 2

Número de pacientes, segundo o resultado anatomopatológico da peça cirúrgica

\begin{tabular}{l|c|c}
\hline $\begin{array}{l}\text { Anatomopatológico } \\
\text { da peça cirúrgica }\end{array}$ & $\begin{array}{c}\text { No de } \\
\text { pacientes }\end{array}$ & $\%$ \\
\hline Carcinoma papilífero & 13 & 65 \\
Carcinoma folicular & 6 & 30 \\
Carcinoma de células de Hürthle & 1 & 5 \\
\hline
\end{tabular}

Nos demais 15 pacientes, a análise anatomopatológica do lobo contralateral da peça cirúrgica foi negativa em $11(73,3 \%)$ para carcinoma e positiva nos quatro restantes $(26,7 \%)$, sendo três papilíferos e um folicular (Tabela 3).

Em relação às complicações, dois pacientes $(13,3 \%)$ apresentaram disfonia temporária de 15 a 40 dias revertidos com fonoterapia, dois $(13,3 \%)$ de hipoparatireoidismo, sendo um temporário com 35 dias e outro permanente (Tabela 4). Não houve mortalidade.

Tabela 3

Número de pacientes com relação ao exame anatomopatológico da peça cirúrgica da segunda intervenção cirúrgica

\begin{tabular}{l|c|c}
\hline $\begin{array}{l}\text { Anatomopatológico } \\
\text { da peça cirúrgica }\end{array}$ & $\begin{array}{c}\text { No de } \\
\text { pacientes }\end{array}$ & $\%$ \\
\hline Ausência de carcinoma & 11 & 73,3 \\
Carcinoma papilífero & 3 & 20,0 \\
Carcinoma folicular & 1 & 6,7 \\
\hline
\end{tabular}

Tabela 4

Número de pacientes com relação às complicações pós-operatórias

\begin{tabular}{l|c|r}
\hline Complicações & $\begin{array}{c}\text { No de } \\
\text { pacientes }\end{array}$ & $\%$ \\
\hline Disfonia temporária & 2 & 13,3 \\
Hipoparatireoidismo temporário & 1 & 6,6 \\
Hipoparatireoidismo permanente & 1 & 6,6 \\
\hline
\end{tabular}

\section{DISCUSSÃO}

A maioria das séries estudadas nos últimos anos tem preconizado a tireoidectomia total para o tratamento cirúrgico do carcinoma diferenciado da tireóide por esse procedimento oferecer várias vantagens como facilidade de detecção e ablação de metástase à distância pela radioiodoterapia, maior sensibilidade do nível de tireoglobulina para avaliação de doença recorrente, principalmente a distância, pre- 
venção da possibilidade de carcinoma diferenciado da tireóide residual se degenerar em anaplásico e maior sobrevida com diminuição da taxa de recorrência ${ }^{3,5,7,8,10,11,15,18-}$ 21,23 . Assim, se justifica nos pacientes que foram submetidos à operação inicial apenas lobectomia com istmectomia, por terem suspeita diagnóstica de doença benigna, que seja realizada na reoperação a complementação da tireoidectomia.

Algumas séries têm demonstrado que a possibilidade de apresentar tumor residual no lobo contralateral é variável de 21 a $62 \%$, quando é realizada a totalização da tireoidectomia de modo profilático ${ }^{8,15,18,20,24}$. Esses dados são compatíveis com a série estudada por nós, que foi de $26,6 \%$.

Apesar de essa incidência não ser desprezível, alguns autores ainda têm contestado a necessidade de totalização da tireoidectomia por acharem que o tumor, quando presente no lobo contralateral, geralmente não tem significância clínica maior do que $5 \%$, além de que o tamanho do tumor primário e o envolvimento linfonodal não são indicativos de doença bilateral ${ }^{8,11}$. No entanto, na nossa série, os quatro pacientes com carcinoma presente no lobo contralateral na complementação da tireoidectomia apresentaram na peça da primeira intervenção operatória tumores maiores do que $2,5 \mathrm{~cm}$.

Já a presença de multicentricidade no lobo primário parece estar associada à maior incidência de carcinoma bilateral, como tem sido demonstrado por outros autores ${ }^{6,25}$. Embora essa assertiva possa ser verdadeira, em nenhum dos nossos pacientes estudados a lesão no lobo primário foi multifocal. Desde que tem sido demonstrado que a incidência de carcinoma latente pode ser elevada, a questão que ainda permanece é: qual método seria mais seguro para remover o lobo contralateral remanescente sem ocasionar morbidade expressiva? Assim, alguns autores, por acharem que a reoperação possa estar associada à maior morbidade, têm preconizado a ablação com iodo radioativo do tecido tireoidiano remanescente em substituição à tireoidectomia total ${ }^{26}$. Entretanto, esse procedimento é contestado por muitos por oferecer algumas desvantagens, como a necessidade de dose de iodo radioativo excessiva, desenvolvimento a longo prazo de adenoma de paratireóide e efeito teratogênico em mulheres jovens em fase de reprodução ${ }^{8,27,28,29}$. Assim, a totalização da tireoidectomia tem, mais uma vez, a sua importância e, se realizada por equipe experiente, proporciona baixa morbidade. Das complicações citadas, o comprometimento do nervo laríngeo recorrente, com conseqüente disfonia, tem sido encontrada em uma incidência de 0 a 5\% 5, 8, 10,15, 18, 20 . Entretanto, na maioria desses casos, a disfonia se apresenta como transitória, como ocorreu na nossa série. Como se observa na Tabela 4, embora a incidência dessa complicação tenha sido maior do que a média esperada na literatura, os dois pacientes recuperaram a integridade da voz após 15 e 40 dias de pósoperatório, sem demonstrar qualquer seqüela na prega vocal, confirmado pela realização de laringoscopia direta.

Outra complicação que tem chamado a atenção é o hipoparatireoidismo, que na nossa série esteve presente em $13,3 \%$ dos casos, compatível com a literatura, que varia de 0 a $16,8 \%$ 3, 5, 8, 10,15, 20. Como se observa na Tabela 4 , em um caso, essa complicação foi temporária, sendo revertida após 35 dias de terapêutica específica, fato esse também demonstrado por outros autores $5,8,15$.

Embora com uma incidência que não ultrapassa a 3\%, alguns autores, mesmo favoráveis à totalização da tireoidectomia, têm preconizado a interrupção do ato cirúrgico quando há muita fibrose no campo operatório para evitar complicações ${ }^{15,18}$. Esse fato ocorreu com um dos nossos pacientes, sendo que não houve oportunidade de acompanhamento de sua evolução. Embora alguns autores indiquem que a totalização da tireoidectomia seja realizada entre três e seis meses da primeira intervenção cirúrgica para prevenir complicações ${ }^{15}$, isto não é o que preconizamos, já que quanto mais precoce for a segunda operação, diminui-se a possibilidade de disseminação hematogênica e linfática nos pacientes com tumor residual no lobo contralateral. Todos os casos estudados foram operados nos primeiros 30 dias de pós-operatório da primeira intervenção, fato este corroborado por outros $5,8,18,20$.

Assim, concluímos que a totalização da tireoidectomia é um procedimento importante no tratamento do carcinoma diferenciado da tireóide pela possibilidade de comprometimento contralateral em percentuais não desprezíveis, além da baixa incidência de morbidade.

\begin{abstract}
Background: Our goal is to analyze tumor invasion of the contralateral thyroid lobe, evaluating the relationship between risk/benefit with complications of a second surgery. Method: From October, 1993 to December, 1996 twenty patients with thyroid tumors were operated. The analysed variables were sex, age, type of surgery, complications, histophatologic examination ot the surgical especimen and contralateral lobe invasion. There were 2 men and 18 women; the age ranged from 17 to 89 years old; the most common histologic type was papillary carcinoma (13 cases) followed by follicular carcinoma (6 cases) and Hürtle cell carcinoma (1 case). The initial surgical procedures were 11 lobectomies plus isthmectomies; 4 lobectomies; 3 subtotal thyroidectomies; 1 total thyroidectomy and 1 isthmectomy. Five patients were not submitted to total thyroidectomy (1 by fibrosis, 3 by loose of follow-up and 1 case of microcarcinoma). Results: Analysis of the contralateral lobe (performed in 15 patients) showed that 11 were negative and 4 positive (26,6\%). Complications observed were dysphonia ( 2 cases treated with fonotherapy), and hypoparathyroidism (1 transient and 1 permanent). Conclusions: The totalization of thyroidectomy is an important procedure for the treatment of malignant tumors of thyroid due by the high rate of contralateral metastasis (26,6\%). Moreover, it's a procedure without mortality with lower complications.
\end{abstract}

Key Words: Thyroid carcinoma, Surgery. 


\section{REFERÊNCIAS}

1. Cady B, Sedgqwick CE, Meissner WA et al. Changing clinical, pathological, therapeutic and survival patterns in differentiated thyroid cancer. Ann. Surg., 1976, 184: 541-546.

2. Cady B, Sedgqwick CE, Meissner WA. Risk factor analysis in differentiated thyroid cancer. Cancer, 1979, 43: 810-820.

3. Clark $\mathrm{OH}$. The treatment of choice for patients with differentiated thyroid cancer. Ann. Surg., 1982, 196: 361-370.

4. Farrar WB, Cooperman M, James AG. Surgical management of papillary and follicular carcinoma of the thyroid. Ann. Surg., 1980, 192: 701-704.

5. Harness JK, Fung L, Thompson MM, et al. Total thyroidectomy: complications and technique. World J. Surg., 1986, 10: 781-786.

6. Logerfo P, Chabot J, Gazatas P. The intraoperative incidence of detectable bilateral and multicentric disease in papillary cancer. Surgery, 1989, 108: 958-965.

7. Noguchi M, Katev N, Miyazaki I.. Controversies in the surgical management of differentiated thyroid carcinoma. Int. Surg., 1996, 81(2): 163-167.

8. Pasieka LJ, Thompson NW, McLeod MK, et al. The incidence of bilateral well differentiated thyroid cancer found at completion thyroidectomy. World J. Surg., 1992, 16: 711-717.

9. Rose RG, Kelsey MP, Russell RA. Follow-up of thyroid cancer treated by unilateral lobectomy. Am. J. Surg., 1963, 106: 494-500.

10. Sand J, Palkola K, Salmi J. Surgical complication after total thyroidectomy and resections for differentiated thyroid carcinoma. Ann. Chir. Gynaecol., 1996, 85: 305-308.

11. Tollefsen HR, Shah JP, Huvos AG. Papillary carcinoma of the thyroid. Recorrence in the thyroid gland after initial surgical treatment. Am. J. Surg., 1972, 124: 468-472.

12. Wanebo HJ, Andrews W, Kaiser DL. Thyroid cancer. Some basic considerations. Am. J. Surg., 1981, 142: 474-479.

13. Brooks JR, Starnes F, Brooks DC, et al. Surgical therapy for thyroid carcinoma: a review os 1249 solitary thyroid nodules. Surgery, 1988, 104: 940-945.

14. Grant CS, Hay ID, Gough, IR, et al. Local recurrence in papillary thyroid carcinoma: is extent of surgical resection important? Surgery, 1988, 104: 954-959.

15. De Jong AS, Demeter JG, Lawrence NA et al. Necessity and safety of completion thyroidectomy for differentiated thyroid carcinoma. Surgery, 1992, 112: 734-739.

16. Hamburguer JI, Hamburger SW. Declining role of frosen section in surgical plannning for thyroid nodules. Surgery, 1985, 2: 307-312.

17. Miller JM, Kni SR, Hamburguer JI. The diagnosis of malignant follicular neoplasms of the thyroid by needle biopsy. Cancer, 1985, 55: 2812-2817.

18. De Groot LJ, Kaplan EL. Second operations for "completion" of thyroidectomy intreatment of differentiated thyroid cancer. Surgery, 1991, 110: 936-940.
19. Hoie J, Stenwing E, Brennhoud IO. Surgery in papillary thyroid carcinoma: a review of 730 patients. J. Surg. Oncol., 1988, 37: 147-151.

20. Scheumann GFW, Seeligwe H, Mushott TJ, et al. Completion thyroidectomy in 131 patients with differentiated thyroid carcinoma. Eur. J. Surg., 1996, 162: 677-684.

21. Ward PH. The surgical treatment of thyroid cancer. Arch. Otolaryngol. Head Neck Surg., 1986, 12: 1204-1208.

22. Beahrs $\mathrm{OH}$, Vandertoll DS Complication of secondary thyroidectomy. Surg. Gynecol. Obstet., 1963, 117: 535-541.

23. Girelli ME, Busnardo B, Amerio R, et al. Serum thyroglobulin level in patients with well-differentiated thyroid cancer during suppression therapy: study on 429 patients. Eur. J. Nucl. Med., 1985, 10: 252-256.

24. Rossi RL, Cady B, Silvermann ML, et al. Current results of conservative surgery for differentiated thyroid carcinoma. World J. Surg, 1986, 10: 612-617.

25. Calabro S, Auguste L, Altie JN. Morbidity of completion thyroidectomy for initially misdiagnosed carcinoma. Head \& Neck Surg., 1988, 10: 235-239.

26. Goolden AWG. The indications for ablating normal thyroid tissue with I-131 in differentiated thyroid cancer. Clin. Endocrinol., 1985, 23: 81-85.

27. Beierwaltes WH, Rabbani R, Dmuchowski C, et al. An analysis of "Ablation of thyroid remnants" with I-131 in 511 patients from 1947-1984: Experience at the University of Michigan. J. Nucl. Med., 1984, 25: 12801286.

28. Bonderson AG, Bonderson L, Thompson NW. Hyperpathyroidism after treatment with radioactive iodine: not only a coincidence? Surgery, 1989, 106: 1025-1029.

29. Ramacciotti C, Pretorius HT, Line BR, et al. Ablation of non malignant thyroid remnants with low doses of radioactive iodine: concise communication. $\mathrm{J}$. Nucl. Med., 1982, 23: 483-485.

Endereço para correspondência:

Dr. José Luiz Braga de Aquino

Rua Cel. Quirino, 1636/03

13025-002 — Campinas-SP 